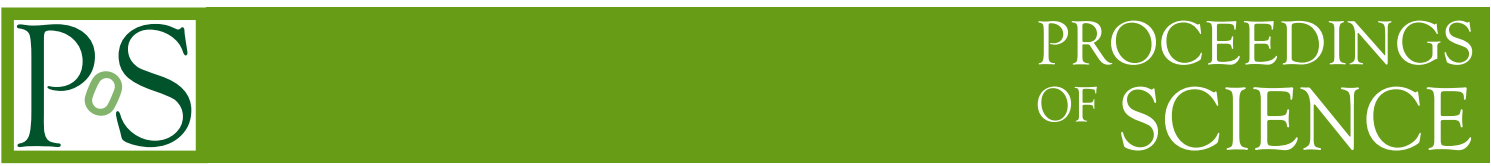

\title{
Anatomy of a deformed symmetry: field quantization on curved momentum space
}

\author{
Michele Arzano* \\ Institute for Theoretical Physics, \\ Utrecht University, \\ Leuvenlaan 4, Utrecht 3584 CE, The Netherlands \\ E-mail: arzano@uu.nl
}

\begin{abstract}
We discuss free field quantization in theories with momentum space given by a non-abelian group manifold. This latter feature is encountered in the description of relativistic particles coupled to three dimensional gravity, a fact which motivates the investigation of such models in four dimensions. Starting with the construction of a one-particle Hilbert space from a classical $\kappa$-deformed phase space (a well studied four-dimensional model of relativistic kinematics with group valued momenta) we show how curvature in momentum space leads to an ambiguity in the quantization procedure reminiscent of the ambiguities encountered for quantum fields in curved space-times. Moving to the multiparticle sector I will discuss how the non-abelian composition of field modes induces additional structure in the Fock space inducing a non-trivial, momentum-dependent statistics. This richer structure allows for the possibility of entanglement between the field modes and "planckian" degrees of freedom.
\end{abstract}

Corfu Summer Institute on Elementary Particles and Physics - Workshop on Non Commutative Field Theory and Gravity,

September 8-12, 2010

Corfu Greece

\footnotetext{
*Speaker.
} 


\section{Introduction}

From a quantum field theoretic point of view an "elementary particle" is an irreducible representation of the Poincaré group. In scattering experiments multiparticle states are defined by collections (symmetrized tensor products) of such representations. It is clear that if one wants to handle a quantum field theory (QFT) (even a trivial non-interacting one) in a generic curved space the lack of global Poincaré symmetries renders the definition of particle cumbersome [1]. In spacetimes with enough symmetry however one can still construct "asymptotic" particle states but in this case different observer might disagree on the particle content of a quantum state. This fundamental observation lies at the basis of the celebrated Hawking effect.

A fact that has been appreciated relatively recently about certain types of non-commutative field theories is that their formulation in momentum space leads to a field theory living on a Lie group (see [2] and references therein).

Field theories on momentum group manifolds, although exhibiting highly non-trivial mathematical structure possess several nice features. Indeed the curvature in momentum space introduces a natural cut off scale which is compatible with relativistic transformations which are now determined by the action of the Lorentz group on the (portion of) homogeneous space describing the group manifold (see e.g. [3] for details).

Interestingly enough curved momenta are encountered in three dimensional gravity where due to the topological nature of the theory particles need to be introduced as defects in the space-time manifold $[4,5,6]$. This suggested speculations that this type of field theories could be relevant for regimes of semiclassical gravity when the local degrees of freedom of the gravitational field can be neglected $[7,8]$.

In this talk I review the basics of quantization of a relativistic particle with momentum living on a Lie group taking as a "case study" the momentum sector of the $\kappa$-Poincaré algebra [9]. After a quick discussion on the relation between the phase space of classical relativistic particle and that of a Klein-Gordon field I recall the steps that lead from the latter to the Hilbert space of a QFT. As in the case of QFT in curved space-times a free quantum field with curved momentum space manifests interesting features. On one side in quantizing the theory one encounters an ambiguity in the definition of a "one-particle" Hilbert space due to the lack of a preferred set of coordinates on the momentum group manifold [2]. At the multi-particle level the non-abelian composition law for the field modes, induced by the non-trivial group structure of momenta, leads to a "momentumshifting" symmetrization $[10,11,12,13,14]$. The latter will be responsible for a Planckian "fine structure" of the Fock space [15]. I will conclude by briefly discussing some interesting consequences that the non-trivial Fock space structures might have for entanglement and decoherence.

\section{From particles to (quantum) fields}

The most general description of the phase space of a classical relativistic point particle is given by a construction known as co-adjoint orbit [16]. This formulation is alternative to the usual one in terms of cotangent bundle of a configuration space and it is particularly useful for systems possessing symmetries. In the present context this description is advantageous since for theories defined on a "non-commutative space-time" the operational description of configuration space is 
not straightforward (and recently subject of debate, see e.g. [17, 18] and references therein).

We consider a classical mechanical system which admits a continuous group of symmetries $G$. We take its phase space manifold to be given by the orbit of the symmetry group $G$ on the dual space $\mathfrak{g}^{*}$ of its Lie algebra $\mathfrak{g}$. Since $G$ acts via its co-adjoint representation this is what we call the "coadjoint orbit". On this manifold the symplectic structure is given by the natural Poisson bracket on functions on $\mathfrak{g}^{*}$ given

$$
\{f, g\}(Y) \equiv\left\langle Y,\left[(d f)_{Y},(d g)_{Y}\right]\right\rangle,
$$

where $(d f)_{Y},(d g)_{Y} \in\left(\mathfrak{g}^{*}\right)^{*} \simeq \mathfrak{g}$ and $\langle Y, \xi\rangle \equiv Y(\xi)$ is the natural pairing of dual vector spaces. For a relativistic point particle we specialize to $G$ being the Poincaré group $I S O(3,1)=S O(3,1) \ltimes \mathbb{R}^{3,1}$ and thus $\mathfrak{g}^{*}=\mathfrak{i s o} \mathfrak{o}^{*}(3,1) \equiv \mathfrak{s o}^{*}(3,1) \oplus\left(\mathbb{R}^{3,1}\right)^{*}$. Co-adjoint orbits $\mathscr{O}_{m, s}$ are given by hyper-surfaces on iso* $(3,1)$ determined by two Casimir functions labelled by the mass and spin of the particle (see [19] for details). We are ultimately interested in the quantization of a scalar field so we focus on a spinless particle. The "momentum sector" of its phase space manifold $\mathscr{O}_{m, 0}$ is obtained from the restriction of the action of the Lorentz group to the dual space $\mathfrak{t}^{*} \subset \mathfrak{i s o}^{*}(3,1)$ and it is given by the mass shell $M_{m} \subset \mathfrak{t}^{*} \equiv \mathbb{R}^{3,1}$

$$
\mathscr{C}_{1}(p)=\left(p^{0}\right)^{2}-\mathbf{p}^{2}=m^{2} .
$$

From its definition as a orbit of a symmetry group $M_{m}$ has a natural structure of a homogeneous space, indeed $M_{m} \simeq S O(3,1) / S O(3)$. As a homogeneous space $M_{m}$ admits an invariant measure on its space of functions $C^{\infty}\left(M_{m}\right)$ which can be written as a " $\delta$-measure"

$$
d \mu_{m}=d V \delta\left(\mathscr{C}_{1}(p)\right),
$$

where $d V$ is the ordinary volume four-form on $\mathbb{R}^{3,1}$. We can also view elements of $C^{\infty}\left(M_{m}\right)$ as distributions on $\left(\mathbb{R}^{3,1}\right)^{*}$ given by

$$
\tilde{\phi}(p)=\delta\left(\mathscr{C}_{1}(p)\right) \tilde{f}(p)
$$

with $\tilde{f}(p) \in C^{\infty}\left(\left(\mathbb{R}^{3,1}\right)^{*}\right)$. A necessary and sufficient condition for a distribution to be of the form above is that

$$
\left(\mathscr{C}_{1}(p)-m^{2}\right) \tilde{\phi}(p)=0
$$

which is nothing but the Fourier transform of the Klein-Gordon equation. Notice also that the measure $d \mu_{m}$ allows us to define an invariant inner product

$$
\left(\phi_{1}, \phi_{2}\right)=i \int d^{4} k \delta\left(k^{2}-m^{2}\right) \bar{\phi}_{1}(k) \phi_{2}(k),
$$

which in turn is related via Fourier transform of the field to the Wronskian of the Klein-Gordon equation

$$
\omega\left(\phi_{1}, \phi_{2}\right)=\int_{\Sigma}\left(\phi_{2} \nabla_{\mu} \phi_{1}-\phi_{1} \nabla_{\mu} \phi_{2}\right) d \Sigma^{\mu}
$$

via the relation $\left(\phi_{1}, \phi_{2}\right)=-i \omega\left(\bar{\phi}_{1}, \phi_{2}\right)$. It turns out that the space of solutions to the Klein Gordon equation $\mathscr{S}$, equipped with the symplectic structure given by the Wronskian above provides a "covariant" description of the phase space of a classical field [20].

In order to discuss field quantization let us consider the complexified space of solutions $\mathscr{S}^{\mathbb{C}} \equiv$ $\mathscr{S} \otimes \mathbb{C}$. The key step $[21,22]$ in building a "one-particle" Hilbert space is the introduction of a 
complex structure $J$ on $\mathscr{S}$ (and $\mathscr{S}^{\mathbb{C}}$ ) i.e. a map $J: \mathscr{S} \rightarrow \mathscr{S}$ such that $J^{2}=-1$. The introduction of $J$ gives rise to a decomposition $\mathscr{S}^{\mathbb{C}}=\mathscr{S}^{\mathbb{C}+} \oplus \mathscr{S}^{\mathbb{C}-}$ into two subspaces, $\mathscr{S}^{\mathbb{C}+}$ and $\mathscr{S}^{\mathbb{C}-}$ spanned, respectively, by the eigenvectors of $J$ with eigenvalues $\pm i$ i.e. $J\left(\phi^{ \pm}\right)= \pm i\left(\phi^{ \pm}\right)$. To this rather abstract construction can be given an intuitive physical interpretation. If the background spacetime is static using the timelike Killing vector $\mathscr{L}_{t}$ one can decompose any real solution $\phi \in \mathscr{S}$ into normal modes (e.g. plane waves) of positive and negative energy components with respect to $\mathscr{L}_{t}$

$$
\phi=\phi^{+}+\phi^{-} .
$$

Then the map $J=-\left(-\mathscr{L}_{t} \mathscr{L}_{t}\right)^{-1 / 2} \mathscr{L}_{t}$ is such that

$$
J \phi=i \phi^{+}+(-i) \phi^{-},
$$

i.e. $J$ is a complex structure on $\mathscr{S}$ and it provides a decomposition of $\mathscr{S}^{\mathbb{C}}$ in positive and negative energy subspaces. Such decomposition is crucial in the constructions of the Hilbert space because it allows to define a positive definite inner product starting from the symplectic product $\omega$. Indeed looking at the discussion above it would seem that functions on $M_{m}$ together with the natural inner product 2.6 would already provide us with a Hilbert space. However $M_{m}=M_{m}^{+} \cup M_{m}^{-}$is a twosheeted hyperboloid consisting of positive $M_{m}^{+}$and negative mass-shell $M_{m}^{-}$, which via Fourier transform provide the support for positive and negative energy solutions. One can easily check that the inner product 2.6 is not positive definite for functions on $M_{m}$ but it is if we restrict to $M_{m}^{+}$or equivalently to $\mathscr{S}^{\mathbb{C}+}$. Thus the "one-particle" Hilbert space will be given by the space of functions $C^{\infty}\left(M_{m}^{+}\right)$endowed with the inner product 2.6. Starting from the one-particle Hilbert space one can construct the full bosonic Fock space from the direct sum of "symmetrized" $n$-tensor products of the one-particle space $\mathscr{H}$.

Given a "one-particle" observable $\mathscr{O}$, i.e. a self adjoint operator on $\mathscr{H}$, its action on multiparticle states is given by the second quantized operator [23]

$$
d \Gamma(\mathscr{O}) \equiv 1+\mathscr{O}+(\mathscr{O} \otimes 1+1 \otimes \mathscr{O})+(\mathscr{O} \otimes 1 \otimes 1+1 \otimes \mathscr{O} \otimes 1+1 \otimes 1 \otimes \mathscr{O})+\ldots
$$

Such expression is simply telling us that the operator $\mathscr{O}$ acts on multiparticle states as a derivative i.e. following the Leibniz rule. The expression above can be re-written in terms of what mathematicians call coproduct $\Delta \mathscr{O}=\mathscr{O} \otimes 1+1 \otimes \mathscr{O}$ of the operator $\mathscr{O}$

$$
d \Gamma(\mathscr{O}) \equiv 1+\mathscr{O}+\Delta \mathscr{O}+\Delta_{2} \mathscr{O}+\ldots+\Delta_{n} \mathscr{O}+\ldots
$$

where $\Delta_{n} \mathscr{O}=(\Delta \otimes 1) \circ \Delta_{n-1}, \Delta_{1} \equiv \Delta$ with $n \geq 2$. The coproduct encodes information on how observables act on a multi-component quantum system and, in our specific case, on the additivity properties of observables for the multiparticle sector of a quantum field Hilbert space.

We showed that the complex structure $J$ and the coproduct $\Delta$ are ingredients needed to build the Hilbert space of a quantum field from the classical field phase space. The first is needed to single out a notion of positive energy which determines the notion of particle and antiparticle the second is essential in the description of composite system and the observables associated with them. In the following we will see how both such objects play a crucial role when we attempt to quantize fields for which $M_{m}$ is embedded into a group manifold. 


\section{Phase space with group valued momenta}

We saw that at the phase space level we speak of "four-momentum" of a classical relativistic particle referring to coordinates on the space $t^{*}$ dual to the algebra of translation generators. When moving to field theory we essentially work with functions on $t^{*}$ and thus the central objects become plane waves which are "characters" namely elements of the group $T^{*}$ dual to the translation group. Momenta in this case are labels of such waves or coordinates on $T^{*}$. In ordinary relativistic theories $T^{*}$ as a manifold is simply Minkowski and its Lie algebra $\mathfrak{t}^{*}$, as tangent space to the identity, can be identified with $T^{*}$ itself. Thus the two notions of momenta coincide as "global coordinates" on Minkowski space.

What happens if we introduce a non-trivial bracket on $t^{*}$, or, in a suggestive but somewhat misleading analogy with a standard relativistic particle, we introduce "non-commutative space-time coordinates"? The first obvious consequence is that the the group law on $T^{*}$ is now non-abelian, namely we have "group-like" plane waves with a non-abelian composition ${ }^{1}$

$$
e_{p} e_{q} \equiv e_{p \oplus q} \neq e_{q \oplus p} \equiv e_{q} e_{p}
$$

and non-trivial group inversion

$$
\left(e_{p}\right)^{-1} \equiv e_{\ominus p} .
$$

Notice how $\mathfrak{t}^{*}$ as a vector space is still Minkowski space but $T^{*}$ is a group manifold and that the two notions of momenta discussed above no longer coincide! How this new state of affairs affects the quantization procedure, namely the choice of the complex structure $J$ and the co-product $\Delta$, is the principal subject of this talk, before we move on, however, I would like to briefly recall why we should be interested in fields with "group-valued" momenta.

As mentioned in the Introduction the main motivation for looking at non-trivial Lie brackets on $\mathfrak{t}^{*}$ comes from the description of point particles in three dimensional gravity. It is well known that the latter is a topological theory since it does not possess local degrees of freedom and it can be formulated as a Chern-Simons theory [25]. A way to couple point particles to such theory is to introduce punctures on the manifold. The main point is that in order to describe the phase space of these particles/punctures, in particular to construct the Poisson structure, one needs to introduce a $r$-matrix i.e. an element of $\mathfrak{g} \otimes \mathfrak{g}$ where $\mathfrak{g}$ is the Lie algebra of the gauge group. Such $r$-matrix defines a "co-commutator", a map $\delta: \mathfrak{g} \rightarrow \mathfrak{g} \otimes \mathfrak{g}$ which in turns induces an non-trivial Lie-bracket on $\mathfrak{g}^{*}$ (for details of the construction see [26]). In the case of vanishing cosmological constant it has been shown [6] that $\mathfrak{t}^{*} \equiv \mathfrak{s u}(1,1)$ i.e. $T^{*} \equiv S U(1,1)$ and thus momenta, as field modes, i.e. labels of plane waves, are coordinates on a hyperboloid in $\mathbb{R}^{3,1}$.

In four dimensions we find a similar "bending" of momentum space in the translation sector of the $\kappa$-Poincaré algebra. This algebra was initially proposed in the context of quantum deformation of relativistic symmetries with no apparent connection with gravity [9]. A decade after its introduction it was realized [27] that the dual to the algebra of translation generators is the Lie algebra given by

$$
\left[P_{\mu}^{*}, P_{v}^{*}\right]=-\frac{i}{\kappa}\left(P_{\mu}^{*} \delta_{v}^{0}-P_{v}^{*} \delta_{\mu}^{0}\right),
$$

\footnotetext{
${ }^{1}$ Notice how the non-vanishing Lie bracket on $\mathrm{t}^{*}$ does not affect the abelian group structure of $T$, rather it introduces a non-trivial Poisson-Lie structure on the latter (for more details see e.g. [24]).
} 
or identifying the elements of $\mathfrak{t}^{*}$ as space-time coordinates

$$
\left[x_{i}, x_{j}\right]=0,\left[x_{0}, x_{j}\right]=\frac{i}{\kappa} x_{j},
$$

a non-commutative space-time known as $\kappa$-Minkowski space. Momenta are now coordinates on the non-abelian group $T^{*}=B$ obtained by exponentiating the Lie algebra above. As a manifold this group is given by "half" de Sitter space [28]

$$
-\eta_{0}^{2}+\eta_{1}^{2}+\eta_{2}^{2}+\eta_{3}^{2}+\eta_{4}^{2}=\kappa^{2} ; \quad \eta_{0}-\eta_{4}>0
$$

where $\eta_{\mu}$ are coordinates in $\mathbb{R}^{4,1}$, five dimensional Minkowski space in which the hyperboloid is embedded and $\kappa$, related to the "momentum space" cosmological constant, is a UV energy scale which according to the analogy with the three dimensional gravity case it is usually identified with the Planck energy. Let's now look at the non-trivial composition law of the group-valued plane waves. This can be formally obtained using the Baker-Campbell-Hausdorff formula ( see e.g. [29]) but of course the explicit expression for the addition of spatial momenta and energies will depend on the choice of coordinates on the group manifold. Let us consider, for example, the following one-parameter family of group splittings

$$
e_{p} \equiv e^{-i \frac{1-\beta}{2} p^{0} P_{0}^{*}} e^{i p^{j} P_{j}^{*}} e^{-i \frac{1+\beta}{2} p^{0} P_{0}^{*}},
$$

with $0 \leq|\beta| \leq 1$. It can be shown [11,2] that for each different splitting one has the following non-abelian additions of momenta

$$
p \oplus_{\beta} q=\left(p^{0}+q^{0} ; p^{j} e^{\frac{1-\beta}{2 \kappa} q^{0}}+q^{j} e^{-\frac{1+\beta}{2 \kappa} p^{0}}\right),
$$

while the group inversion will reflect in the non-trivial "antipode"

$$
\ominus_{\beta} p=\left(-p^{0} ;-e^{\frac{-\beta}{\kappa}} p^{0} p^{i}\right) .
$$

Fixing a particular set of coordinates on $T^{*}$ we can illustrate other salient features of the deformed $\kappa$-Poincaré algebra. We focus on the case $\beta=1$ corresponding to "flat-slicing" coordinates

$$
\begin{aligned}
& \eta_{0}\left(p_{0}, \mathbf{p}\right)=\kappa \sinh p_{0} / \kappa+\frac{\mathbf{p}^{2}}{2 \kappa} e^{p_{0} / \kappa}, \\
& \eta_{i}\left(p_{0}, \mathbf{p}\right)=-p_{i} e^{p_{0} / \kappa}, \\
& \eta_{4}\left(p_{0}, \mathbf{p}\right)=-\kappa \cosh p_{0} / \kappa-\frac{\mathbf{p}^{2}}{2 \kappa} e^{p_{0} / \kappa} .
\end{aligned}
$$

This "basis" of the $\kappa$-Poincaré algebra was first used in [27] to exhibit the connection of the latter with $\kappa$-Minkowski non-commutative space-time and has been widely used in field theoretic applications $[10,30,31]$. One of the consequences of the curvature of the momentum manifold is that the adjoint action of boosts on translation generators in this basis is non-linear [32],

$$
\left[N_{j}, P_{l}\right]=i \delta_{l j}\left(\frac{\kappa}{2}\left(1-e^{-\frac{2 P_{0}}{\kappa}}\right)+\frac{1}{2 \kappa} \vec{P}^{2}\right)+\frac{i}{\kappa} P_{l} P_{j} .
$$

while rotations act in the usual way. Perhaps the most important aspect that the non-trivial group 
structure of $T^{*}$ introduces is the change in the rule for extending the action of the algebra generators to tensor product representation i.e., according to the discussion at the end of Section the coproduct. Indeed the non-trivial momentum composition law 3.7 for plane waves translates into a non-abelian combination of momenta for a two-particle state. Since this is just a tensor product of irreducible representations of the algebra this behaviour can be recast in terms of a modified co-product for spatial translation generators

$$
\Delta\left(P_{i}\right)=P_{i} \otimes 1+\exp \left(-P_{0} / \kappa\right) \otimes P_{i}
$$

with the ordinary composition rule for energies is encoded in a ordinary ("Leibniz-like") co-product for time translations generators

$$
\Delta\left(P_{0}\right)=P_{0} \otimes 1+1 \otimes P_{0} .
$$

It turns out that the structure of the $\kappa$-Poincare algebra is, in technical terms, that of a Hopf algebra and as such it also determines the way the other generators act on tensor product representations ${ }^{2}$. One has

$$
\begin{aligned}
& \Delta\left(N_{j}\right)=N_{j} \otimes 1+e^{-P_{0} / \kappa} \otimes N_{j}+\frac{\varepsilon_{j k l}}{\kappa} P_{k} \otimes M_{l} \\
& \Delta\left(M_{i}\right)=M_{i} \otimes 1+1 \otimes M_{i},
\end{aligned}
$$

from which we can see that rotations act in the usual way while the action of boost is non-Leibniz as for spatial translations. In the limit of the deformation parameter $\kappa$ going to infinity one recovers the ordinary Poincaré algebra and the Leibniz prescription for the action on tensor products of representations. There remains one important object to discuss at the algebraic level: the invariant Casimir operator. This plays a central role in the context of field theory since it represents the "momentum version" of the d'Alembertian and it determines the mass shell. In the next Section we will discuss the issues that we have to deal with when the mass shell is embedded in a curved manifold and thus there is no preferred set of coordinates for its description in terms of energy and spatial momenta.

\section{A new quantization ambiguity}

As discussed in Section 2 the "one-particle" Hilbert space of a quantum field can be built starting from functions on the mass shell, using the natural invariant measure associated with a homogeneous space to define a inner product on it and finally introducing a complex structure to select a subspace on which the latter is positive definite. While the first two steps can be carried out rather straightforwardly also in the case of a curved momentum space manifold [31] we will see that, unlike the case of ordinary QFT in Minkowski space, in curved momentum space one does not have a preferred notion of "positive energy" or choice of complex structure. In order to show this let us recall that in principle even for ordinary momentum space embedded in $\mathbb{R}^{3,1}$ one could choose a new set of coordinates obtained from non-linear redefinitions of ordinary "cartesian" momenta. These would combine into a non-linear Casimir and change the composition rule of momenta.

\footnotetext{
${ }^{2}$ Notice that also the ordinary Poincaré algebra equipped with the ordinary Leibniz rule action on tensor product representation is a Hopf algebra although trivial.
} 
However in the flat momentum space case one does have a preferred choice of coordinates in momentum space. The are precisely the cartesian coordinates, which correspond to a representation of translations as local symmetry generators $P_{0}, P_{i}$ [33] for which the Casimir is the usual $C_{1}(P)=$ $P_{0}^{2}-\mathbf{P}^{2}$ and

$$
\Delta P_{\mu}=P_{\mu} \otimes 1+1 \otimes P_{\mu},
$$

i.e. we are choosing translation generators which act according to the Leibniz rule. In the algebra of polynomials of generators of a Lie algebra such elements are called "primitive". The complex structure we use to define a notion of positive energy will be, as usual $J=i \frac{P_{0}}{\left|P_{0}\right|}$ from which we can construct a projector on the positive mass-shell $P^{+}=\frac{1}{2}\left(1+\frac{P_{0}}{\left|P_{0}\right|}\right)$. Now we come to our main point. When momentum space is a group manifold there is no preferred choice of complex structure since there are no primitive elements among the polynomials of translation generators [34]. Even if we choose a set of coordinates $P_{0}, P_{i}$ for which the Casimir is the ordinary $C_{1}(P)=P_{0}^{2}-\mathbf{P}^{2}$ we will have a non-Leibniz action of the latter on multiparticle states i.e. no matter what choice of translation generators, their coproduct will always be non symmetric i.e. $\sigma \circ \Delta \neq \Delta$ (where $\sigma(a \otimes b)=b \otimes a)$.

To give a concrete example we consider the flat slicing coordinates of the previous section. The mass Casimir expressed in terms of the translation generators associated to these coordinates will be

$$
\mathscr{C}_{1}^{\kappa}(P)=\left(2 \kappa \sinh \left(\frac{P_{0}}{2 \kappa}\right)\right)^{2}-\mathbf{P}^{2} e^{P_{0} / \kappa} .
$$

For a massless field the Hilbert space will be built from functions on deformed mass shell $M_{m}^{\kappa}$ defined by $\omega_{\kappa}^{ \pm}(\mathbf{p})=-\kappa \log \left(1 \mp \frac{|\mathbf{p}|}{\kappa}\right)$. The time translation generator associated with these coordinates can be used to define a complex structure and a projector which singles out the positive mass-shell. The Hilbert space will be given by $C^{\infty}\left(M_{m}^{\kappa+}\right)$ equipped with inner product

$$
\left(\phi_{1}, \phi_{2}\right)_{\kappa}=\int_{M_{m}^{K+}} \frac{d \mu(\mathbf{p})}{2 \omega_{\kappa}(\mathbf{p})} \bar{\phi}_{1}(\mathbf{p}) \phi_{2}(\mathbf{p})
$$

where $d \mu(\mathbf{p})=\frac{e^{3 p_{0} / \kappa}}{(2 \pi)^{4}} d p_{0} d^{3} \mathbf{p}$ is the Haar measure on momentum group manifold. It is easily checked that for "transplanckian" ( $|\mathbf{k}|>\kappa)$ modes 4.3 is no longer positive definite. Thus to obtain the $\kappa$-one particle Hilbert space it does not suffice to restrict to functions on the positive mass-shell but also to modes whose modulus does not exceed the UV deformation parameter $\kappa$. It is interesting to notice that the presence of such cut-off does not conflict with Lorentz symmetry since it can be shown [32] that under finite boosts constructed from the deformed action 3.10 the modulus of a field mode approaches $\kappa$ as the boost parameter goes to infinity.

Notice that the same construction can be repeated for any other set of coordinates on the momentum group manifold and in particular for the coordinate sets defined by the group splitting 3.6 for various values of the parameter $\beta$. The positive definite inner product will of course not be affected by the change of coordinates in the group, an indication that, unlike the case of quantum fields in curved space-times, all constructions share the same vacuum state. For different choices of momentum coordinates, however, the modes labelling the one particle states will obey different energy-momentum dispersion relations. 
The curvature in momentum space will also introduce important new features in the multiparticle sector of the theory. For simplicity we restrict our discussion to two-particle states. Recall here that due to the indistinguishability of the components of a composite quantum system a twoparticle state of a quantum scalar field will be described by the symmetrized superposition of tensor products of one-particle states

$$
1 / \sqrt{2}\left(\left|\mathbf{p}_{1}\right\rangle \otimes\left|\mathbf{p}_{2}\right\rangle+\left|\mathbf{p}_{2}\right\rangle \otimes\left|\mathbf{p}_{1}\right\rangle\right) .
$$

If we try to extend the same construction to the deformed case we soon run into troubles. Indeed due to the non-symmetric nature of the co-product for any choice of coordinates it is easy to see that the state 4.4 is not an eigenstate of the generators of linear translations $P_{i}$. It turns out $[10,11]$ that one has to resort to a "momentum-dependent" symmetrization i.e., again working in flat-slicing coordinates, the usual flip operator $\sigma\left(\left|\mathbf{p}_{1}\right\rangle \otimes\left|\mathbf{p}_{2}\right\rangle\right)=\left(\left|\mathbf{p}_{2}\right\rangle \otimes\left|\mathbf{p}_{1}\right\rangle\right)$ will be replaced by

$$
\sigma^{K}\left(\left|\mathbf{p}_{1}\right\rangle \otimes\left|\mathbf{p}_{2}\right\rangle\right)=\left|\left(1-\varepsilon_{1}\right) \mathbf{p}_{2}\right\rangle \otimes\left|\left(1-\varepsilon_{2}\right)^{-1} \mathbf{p}_{1}\right\rangle,
$$

with $\varepsilon_{i}=\frac{\left|\mathbf{p}_{i}\right|}{\kappa}$. Given two one-particle modes $\mathbf{p}_{1}$ and $\mathbf{p}_{2}$ one can obtain two different two-particle states

$$
\begin{aligned}
& \left|\mathbf{p}_{1} \mathbf{p}_{2}\right\rangle_{\kappa}=\frac{1}{\sqrt{2}}\left[\left|\mathbf{p}_{1}\right\rangle \otimes\left|\mathbf{p}_{2}\right\rangle+\left|\left(1-\varepsilon_{1}\right) \mathbf{p}_{2}\right\rangle \otimes\left|\left(1-\varepsilon_{2}\right)^{-1} \mathbf{p}_{1}\right\rangle\right] \\
& \left|\mathbf{p}_{2} \mathbf{p}_{1}\right\rangle_{\kappa}=\frac{1}{\sqrt{2}}\left[\left|\mathbf{p}_{2}\right\rangle \otimes\left|\mathbf{p}_{1}\right\rangle+\left|\left(1-\varepsilon_{2}\right) \mathbf{p}_{1}\right\rangle \otimes\left|\left(1-\varepsilon_{1}\right)^{-1} \mathbf{p}_{2}\right\rangle\right]
\end{aligned}
$$

These states are different because according to the non-symmetric form of the coproduct they will carry different linear momenta

$$
\begin{aligned}
& \mathbf{P}_{12}=\mathbf{p}_{1} \oplus \mathbf{p}_{2}=\mathbf{p}_{1}+\left(1-\varepsilon_{1}\right) \mathbf{p}_{2} \\
& \mathbf{P}_{21}=\mathbf{p}_{2} \oplus \mathbf{p}_{1}=\mathbf{p}_{2}+\left(1-\varepsilon_{2}\right) \mathbf{p}_{1}
\end{aligned}
$$

where, specializing 3.7 to $\beta=1, \mathbf{p} \oplus \mathbf{q} \equiv \mathbf{p}+e^{-\omega_{\mathbf{p}} / \kappa} \mathbf{q}$. One should also notice that, due to the undeformed coproduct for time translation generators the two states will be degenerate in energy, $E_{12}=E_{21}$.

In general starting from $n$ different one-particle modes there will be $n$ ! corresponding $n$-particle states each associated with a different eigenvalue of the spatial translations obtained by a permutation of the addenda in the non-abelian sum. Of course this pattern will occur for any choice of coordinates and it can be shown [11] that the symmetrization itself is independent of the particular coordinates adopted. What does depend on the choice of coordinates, as one might expect from the discussion above, is the way the field modes combine, e.g. for two-particle states it follows directly from 3.7 that the modes will combine to give a different total momentum according to the value of the parameter $\beta$.

To summarize: in order to quantize a classical field theory whose modes parametrize group valued plane waves one needs to pick a set of coordinates on the group manifold. In ordinary field theory where the mass-shell is embedded in Minkowski space there exists a preferred choice of coordinates, the usual cartesian momenta, for which we have a trivial (Leibniz-like) co-product. In our case there is no preferred choice of coordinates on the momentum manifold, since there is no set of coordinates for which one has a trivial co-product. Different choices of coordinates will correspond 
to Hilbert spaces whose base states are labelled by modes which obey different dispersion relations and composition laws in the multiparticle sector. The interesting question at this point is whether there exist some kind of physical input which could dictate a preferred choice of coordinates on momentum space. This important point will be addresses in future work.

\section{Hidden entanglement at the Planck scale}

A deeper look at the multiparticle space construction sketched above reveals that the non-trivial algebraic structure introduced by having group valued momenta endows the Fock space with a "fine structure". Indeed the various multiparticle states one can construct from a set of one-particle kets (which in the absence of deformation would be degenerate) can in principle be distinguished by measuring their momentum splitting. For example for the two particle states above the momentum splitting is given by

$$
\left|\Delta \mathbf{P}_{12}\right| \equiv\left|\mathbf{P}_{12}-\mathbf{P}_{21}\right|=\frac{1}{\kappa}\left|\mathbf{p}_{1}\right| \mathbf{p}_{2}\left|-\mathbf{p}_{2}\right| \mathbf{p}_{1}|| \leq \frac{2}{\kappa}\left|\mathbf{p}_{1}\right|\left|\mathbf{p}_{2}\right|
$$

which is of order $\left|\mathbf{p}_{i}\right|^{2} / \kappa$. In other words the 2-particle Hilbert (sub)space becomes $\mathscr{H}_{\kappa}^{2} \cong \mathscr{S}_{2} \mathscr{H}^{2} \otimes$ $\mathbf{C}^{2}$, where $\mathscr{I}_{2} \mathscr{H}^{2}$ is the ordinary symmetrized two-particle Hilbert space. Indeed keeping in mind that the two states are degenerate in energy we can write them as

$$
\begin{aligned}
& |E\rangle \otimes|\uparrow\rangle=\left|\mathbf{p}_{1} \mathbf{p}_{2}\right\rangle_{\kappa} \\
& |E\rangle \otimes|\downarrow\rangle=\left|\mathbf{p}_{2} \mathbf{p}_{1}\right\rangle_{\kappa}
\end{aligned}
$$

with $E \equiv E_{12}=E_{21}=\omega\left(\mathbf{p}_{1}\right)+\omega\left(\mathbf{p}_{2}\right)$. Due to this additional structure we can now have states in which the "macroscopic" degrees of freedom of $S_{2} \mathscr{H}^{2}$ can be entagled with the "planckian" degrees of freedom $\mathbf{C}^{2}$. For example, the following state, superposition of two-particle states with total energies $E_{A}=\omega\left(\mathbf{p}_{1 A}\right)+\omega\left(\mathbf{k}_{2 A}\right)$ and $E_{B}=\omega\left(\mathbf{p}_{1 B}\right)+\omega\left(\mathbf{k}_{2 B}\right)$

$$
|\Psi\rangle=1 / \sqrt{2}\left(\left|E_{A}\right\rangle \otimes|\uparrow\rangle+\left|E_{B}\right\rangle \otimes|\downarrow\rangle\right),
$$

exhibits this type of entanglement. The remarkable fact is that the possibility of this micro-macro entanglement renders possible interesting phenomena of decoherence [15]. To illustrate this consider the unitary evolution of a quantum system $\rho(t)=U(t) \rho(0) U^{\dagger}(t)$. We start with a pure state $\rho(0)$ factorized with respect to the bipartition "macro-micro". If $U(t)$ acts as an "entangling gate" for the macro-micro degrees of freedom, the state $\rho(t)$ will be entangled. A macroscopic observer, not able to resolve the planckian degrees of freedom, at the beginning will see the reduced system in a pure state $\rho_{\text {obs }}(0)=\operatorname{Tr}_{P l} \rho(0)$ since she will be tracing over the "planckian" degrees of freedom. As the system evolves she will see the system in the mixed state

$$
\rho_{\text {obs }}(t)=\operatorname{Tr}_{P l} \rho(t)=\operatorname{Tr}_{P l}\left[U(t) \rho(0) U^{\dagger}(t)\right],
$$

since she is now tracing over degrees of freedom which are entangled. In other words for the macroscopic observer, the evolution is not unitary! This shows how Fock space built out of modes living on a group manifold provides a simple example of a quantum system which exhibits decoherence due to the presence of hidden "planckian" degrees of freedom. Interestingly enough this type of decoherence has been discussed in other contexts as a possible probe for experimental signatures of quantum gravity (see e.g. [35]). Could this open a "phenomenological window" on the deformed QFT models discussed in this talk? 


\section{Summary}

We discussed a model of "deformed" relativistic kinematics where the dual space of the Lie algebra of translation generators is endowed with a non-trivial Lie-bracket or, loosely speaking, in which the system exhibit a "non-commutative configuration space". In the corresponding field theory the new structure leads to plane waves which are element of a Lie group and thus to a "curved momentum space". At the symmetry level one finds a deformed action of the Lorentz algebra which includes an energy scale $\kappa$ related to the curvature of the momentum manifold. Motivations to look at these type of models come from three dimensional gravity [ ], in which the phase space of a point particle exhibit similar structures, and from non-commutative field theory of the "Lie algebra-type" where the Fourier expansion of non-commutative fields leads to group-valued plane waves.

We analyzed in detail the quantization of free field theories with group valued momenta and showed how one encounters ambiguities related to the possibility of choosing different set of coordinate on the momentum manifold. We showed how for a particular choice of coordinates the construction of the one-particle Hilbert space requires, besides the usual restriction to positive energy subspace of the classical field phase space, a "Lorentz invariant" truncation to solutions whose modes "magnitude" is bounded from above by the UV scale $\kappa$. At the multiparticle level the non-trivial behaviour of field modes requires a "momentum-dependent" symmetrization in the construction of bosonic states. Moreover the non-abelian composition rule of field modes is associated to a fine structure of the Fock space accessible only to observers whose detectors have "planckian" resolution. We concluded with some speculation on multiparticle states with planckian-macroscopic entanglement and the possible role that decoherence phenomena associated with such states might have for phenomenologically relevant predictions.

\section{References}

[1] P. C. W. Davies, "Particles Do Not Exist," In *Christensen, S.M. ( Ed.): Quantum Theory Of Gravity*, 66-77, Adam Hilger Ltd. Bristol, 1984

[2] M. Arzano, Phys. Rev. D 83, 025025 (2011) [arXiv:1009.1097 [hep-th]].

[3] M. Arzano, J. Kowalski-Glikman and A. Walkus, Class. Quant. Grav. 27, 025012 (2010) [arXiv:0908.1974 [hep-th]].

[4] G. ’t Hooft, Class. Quant. Grav. 13, 1023 (1996) [arXiv:gr-qc/9601014].

[5] H. J. Matschull and M. Welling, Class. Quant. Grav. 15, 2981 (1998) [arXiv:gr-qc/9708054].

[6] F. A. Bais, N. M. Muller and B. J. Schroers, Nucl. Phys. B 640, 3 (2002) [arXiv:hep-th/0205021].

[7] G. Amelino-Camelia, L. Smolin, A. Starodubtsev, Class. Quant. Grav. 21, 3095-3110 (2004). [hep-th/0306134].

[8] L. Freidel, J. Kowalski-Glikman, L. Smolin, Phys. Rev. D69, 044001 (2004). [hep-th/0307085].

[9] J. Lukierski, A. Nowicki and H. Ruegg, Phys. Lett. B 293, 344 (1992).

[10] M. Arzano and A. Marciano, Phys. Rev. D 76, 125005 (2007) [arXiv:0707.1329 [hep-th]].

[11] M. Arzano and D. Benedetti, Int. J. Mod. Phys. A 24, 4623 (2009) [arXiv:0809.0889 [hep-th]]. 
[12] T. R. Govindarajan, K. S. Gupta, E. Harikumar, S. Meljanac and D. Meljanac, Phys. Rev. D 77, 105010 (2008) [arXiv:0802.1576 [hep-th]].

[13] C. A. S. Young and R. Zegers, Nucl. Phys. B 809, 439 (2009) [arXiv:0807.2745 [hep-th]];

[14] M. Arzano, arXiv:0909.4873 [hep-th].

[15] M. Arzano, A. Hamma, S. Severini, Mod. Phys. Lett. A25, 437-445 (2010). [arXiv:0806.2145 [hep-th]].

[16] Kirillov, A.A., Elements of the Theory of Representations, Springer-Verlag, 1976.

[17] G. Amelino-Camelia, L. Freidel, J. Kowalski-Glikman et al., [arXiv:1101.0931 [hep-th]].

[18] M. Arzano and J. Kowalski-Glikman, arXiv:1008.2962 [hep-th].

[19] J. F. Carinena, J. M. Gracia-Bondia and J. C. Varilly, J. Phys. A 23, 901 (1990).

[20] C. Crnkovic, E. Witten, In *Hawking, S.W. (ed.), Israel, W. (ed.): Three hundred years of gravitation*, 676-684 and Preprint - Crnkovic, C. (86,rec.Dec.) 13 p.; C. Crnkovic, Class. Quant. Grav. 5, 1557-1575 (1988).

[21] P. J. M. Bongaarts, "Linear Fields According To I. E. Segal," In *London 1971, Mathematics Of Contemporary Physics*, Academic Publishing, London 1972, 187-208

[22] A. Ashtekar and A. Magnon, Proc. Roy. Soc. Lond. A 346, 375 (1975).

[23] R. P. Geroch, "Mathematical physics," The University of Chicago Press, 1985

[24] V. Chari and A. Pressley, “A Guide To Quantum Groups,” Cambridge, UK: Univ. Pr. (1994) 651 p

[25] E. Witten, Nucl. Phys. B311, 46 (1988).

[26] C. Meusburger, B. J. Schroers, Nucl. Phys. B806, 462-488 (2009). [arXiv:0805.3318 [gr-qc]].

[27] S. Majid and H. Ruegg, Phys. Lett. B 334, 348 (1994) [arXiv:hep-th/9405107].

[28] J. Kowalski-Glikman and S. Nowak, Class. Quant. Grav. 20, 4799 (2003) [arXiv:hep-th/0304101].

[29] P. Kosinski, J. Lukierski and P. Maslanka, Czech. J. Phys. 50, 1283 (2000) [arXiv:hep-th/0009120].

[30] G. Amelino-Camelia and M. Arzano, Phys. Rev. D 65 (2002) 084044 [arXiv:hep-th/0105120].

[31] M. Arzano and A. Marciano, Phys. Rev. D 75, 081701 (2007) [arXiv:hep-th/0701268].

[32] N. R. Bruno, G. Amelino-Camelia, J. Kowalski-Glikman, Phys. Lett. B522, 133-138 (2001). [hep-th/0107039].

[33] J. Lopuszanski, Singapore, Singapore: World Scientific (1991) 373 p.

[34] M. Arzano, Phys. Rev. D 77, 025013 (2008) [arXiv:0710.1083 [hep-th]].

[35] N. E. Mavromatos, J. Phys. Conf. Ser. 171, 012007 (2009). [arXiv:0904.0606 [hep-ph]]. 